\title{
Agricultural Nanotechnology: Applications and Challenges
}

Khursheed Ahmad Wani ${ }^{*}$ and Richa Kothari ${ }^{2}$

${ }^{1}$ Department of Environmental Science, ITM University, Gwalior, Madhya Pradesh, India.

2Department of Chemistry, ITM University, Gwalior, Madhya Pradesh, India.

Received: 2/15/2018; Revised: 2/23/2018; Accepted: 2/28/2018

\begin{abstract}
Agricultural nanotechnology has emerged in the late 1990s and is developed and applied all over the world. However, this technology has not developed so fast in different sectors of agriculture. This has not even found its market to the expected scale but has the potential to improve agricultural production. This agro nanotechnology can be utilized for developing healthy seeds that can improve plant germination, growth, yield, and quality. This technology has the potential to increase the storage period for vegetables and fruits. Organic pesticides and fertilizers can be developed by the proper use of agro nano technology. It has found it's use in genetic engineering and plant breeding. However, the application of nanotechnology provides numerous advantages related to food safety and quality, at the same time it may present a potential risk not only to human health, but can affect animals and the environment as well as nanoparticles may have toxicological effects on biological systems.
\end{abstract}

Key words: Nanoscience, Application, Agriculture, Potential risks.

\section{Introduction}

The shift towards agricultural nanotechnology to search for the solutions of several agricultural and environmental issues like improved varieties and increased productivity has gained momentum during the last few years [Gogos et al., 2012]. A good number of scientists all over the world have patented their work on agricultural nanotechnology. Most of these patents are in the areas of diseases management and crop protection with different objectives. The prominent examples are nanosensors for soil quality detection, nanomagnets for the removal of soil contaminants, nano-composites for the development of smart delivery systems, nanoscale carriers for efficient delivery of fertilizers and pesticides. Agricultural nanotechnology has emerged as a tool to reduce the amount of sprayed chemicals by smart delivery that has decreased the losses with better approach to water and nutrient management. This has also revolutionized plant breeding and genetic transformation. However, there is no obvious use of the agricultural nanotechnology in the industrial sector that can be marketed to generate employment and increase Gross Domestic Product. This is still restricted to academic and small scale industrial sector and has not been promoted by large enterprises [Claudia et al., 2015]. The trend of patenting in agricultural nanotechnology is growing continuously without any potent application to the market [Gogos et al., 2012; Claudia et al., 2015; Sastry et al., 2010].

The application of nanotechnology in the field of agriculture is blended to arrest the climate change since it will decrease the use of synthetic fertilizers

\section{${ }^{*}$ Corresponding Author:}

Dr. Khursheed A. Wani,

Assistant Professor,

Department of Environmental Science,

ITM University, Gwalior, Madhya Pradesh, India.

E-mail: kuurshidevs@gmail.com that in turn will help to reduce Green House Gases like nitrous oxides, but to what extent the nanotechnology will provide us with agro nanofertilizers will decide its fate [Srilatha, 2011].

The introduction of nanotechnology in the field of rhizosphere or plant root zone technology to influence microbial and enzymatic activity may be a critical factor for plant health and crop productivity. The changes in organic acids, hormones, secondary metabolites may encourage growth of specific prokaryotic and eukaryotic microbial community that can be important to the plant and to the overall ecosystem. The alterations can be achieved with agro nanotechnology to enhance uptake, enhance, and inhibit diseases and plant development by the application of nano-bio-fertilizers. Previously, it has been demonstrated that although de dioxyde de cérium $\mathrm{NPCeO}_{2}$ had modest effect on soybean health, rates of nitrogen fixation and levels of nitrogen fixing bacteria within root nodules on the legume [Priester et al., 2012]. The importance of that plant microbe will provide broader ecosystem services in the long run.

The applicability of nano agro fertilizers for crop improvement is due to multisectorial properties like surface area, cation exchange capacity, ion adsorption, complexation etc and the presence of unlimited number of nano particles on the surface. The different types of nano particles have different surface composition with different active sites that have different reactivity with respect to processes such as adsorption and redox reactions that can be 
utilised successfully to synthesise nano particles for the use in agriculture [Shahid et al., 2016].

\section{Applications of Nano-agrotechnology}

The successful application of nanotechnology in agriculture needs greater scientific calculations for its successful implementation at the larger scale. The aim must be focussed to make soils more capable and fertile with greater productivity and better environmental security. The use of nanotechnology must keep the ions available in the soil ecosystem and should not hamper with ion exchange capacity of the soil adsorption desorption and solubility precipitation reactions. The availability of nutrients to plants has to be equilibrium and use of nanotechnology must not deviate the same.

Genetic improvements of crops are an opportunity in agricultural sector by diverting the genes to a target location at cellular level for gene expression to overcome drought stress. This will also help in accurate farming, natural resource management, and detection of pathogens [Wei et al., 2007]. Nanotechnology will solve the problem of fertilizers driving water contamination and climate vulnerability as well. The technique of nanoremediation will reduce the overall costs of cleaning up large contaminated sites and will decrease the clean up time by eliminating the need for treatment and disposal of contaminated sites. The production of nanozeolites could ameliorate the soil acidity as they supply bases and retain smectites kaolinite in a stable phase [Zhang et al., 2010].

The promotion of biological metabolism is believed to promote the crop growth, increase the yield, and improve the quality of many crop products including cereal crops and cash crops can be achieved by nanotechnology devices although it is yet in its infancy stage.

The relevant applications of nanotechnology at small scale or Research \& Development stage are obvious. A better result was achieved with the experiment conducted with the seeds of legumes (cowpea), cabbage (brassica) and cucumber when treated by nano-863. Cowpea seeds showed an increase of $25.8 \%$ than the control group with germination state improved by $30.7 \%$. The treated water with nano-863showed results two times higher than control, the total number of main leaves was $90 \%$ more than that of the control group and the disease resistance of flowers was enhanced. Anjali et al., 2012 synthesized nanocapsules from Azadirachta indica for disease and pest control in plants. Milani et al., 2012 coated different macronutrients fertilizers with zinc oxide to improve nutrients absorption by plants and the delivery of nutrients to specific sites. McMurray et al., 2006 used TiO2 nanoparticles for the photocatalytic degradation of agrochemicals in contaminated waters that helped in the removal of pesticides from water. Vamvakaki et al., 2007 used liposome-based nano-biosensor that are highly sensitive bio-chemical sensors to closely monitor environmental conditions, plant health and growth. Torney et al., 2007 produced Mesoporus silica nanoparticles carrying DNA or RNA that can be delivered to plant cells for their genetic transformation or to trigger defence responses, activated by pathogens.

\section{Future challenges}

The nanotechnology could cause different risks to human body as well that might get triggered by the introduction of nano technology in agriculture. Nurkiewicz et al., 2008 found that the inhalation of nanosized titanium dioxide engineered nanotechnology materials ENM reached systemic circulation in rats, although it was not tested for human skin by Monteiro-Riviere et al., 2007. However, results obtained in the study conducted by Tinkle et al., 2003 showed that latex particles smaller than $1 \mu \mathrm{m}$ penetrate the outer layers of a skin sample during constant flexing. On the other hand, some studies indicate that nanostructured particles could not penetrate healthy, intact skin Tsuji et al., 2006. Nurkiewicz et al., 2008 demonstrated that inhalation exposure of rats to low concentrations of nanosized titanium dioxide exposure in rats enhanced microvascular dysfunction. Radomski et al., 2005 observed that platelet aggregation and vascular thrombosis due to nanoparticles. Hence, It is important to obtain more data about influence of nanomaterials and nanoparticles on blood and vessels, as ENM can potentially induce adverse effects in any organ in the body. The well vascularised organ may efect brain, and may enter cells and organs and interact with metabolism or migrate in the foetus. Similarly other organs that may become negatively affected by nano technology may be human brain and blood vessels Elder et al, 2009.

\section{References}

1. Gogos, Knauer, and Bucheli. "Nanomaterials in plant protection and fertilization: current state, foreseen applications, and research priorities. J. Agric. Food Chem. 60 (2012): 9781—9792.

2. Claudia, Vigani, and Rodríguez-Cerezo. (2015). "Agricultural Nanotechnologies: What are the current possibilities. Nano Today. 10 (2015); 124 127.

3. Sastry, Rashmi and Rao. "Nanotechnology Patents as R\&D Indicators for Disease Management Strategies in Agriculture". J. Intellect. Prop. Rights. 15 (2010): 197-205

4. Srilatha. "Nanotechnology in Agriculture". J Nanomedic Nanotechnol. 2:7(2011):1-2. 
5. Priester, Ge, Mielke, Horst, Moritz, Espinosa, Gelb, Walker, Nisbet, et al., 'Soybean susceptibility to manufactured nanomaterials with evidence for food quality and soil fertility interruption". Proc. Natl. Acad. Sci. USA 109, (2012). E2451-E2456.

6. Shahid, Mohamad, Mohapatra and Gil. "Characteristic Properties of Nanoclays and Characterization of Nanoparticulates and Nanocomposites". Springer Science+Business Media Singapore 2016 M. Jawaid et al., (eds.), Nanoclay Reinforced Polymer Composites, Engineering Materials. (2016): 978-981.

7. Wei, Yamato, Wei, Zhao, Tsumoto, Yoshimura, Ozawa and Chen. "Genetic nanomedicine and tissue engineering”. Med Clin N Am. (2007): 91:889-898. .

8. Zhang, Fan, Sun, Chen, Liu, Zhang "Effect of nanofertilizer on rice growth characteristic". Heilongizang Agric Sci. 8.(2010):50-52 (in Chinese with English abstract)

9. Anjali, Sharma, Mukherjee and Chandrasekaran, "Neem oil (Azadirachta indica) nanoemulsion--a potent larvicidal agent against Culex quinquefasciatus". Pest Manage. Sci. 68. (2012): 158163.

10. Milani, McLaughlin, Samuel. Stacey, Kirby, Ganga, Beak, and Cornelis. "Dissolution Kinetics of Macronutrient Fertilizers Coated with Manufactured Zinc Oxide Nanoparticles". J. Agric. Food Chem. 60: (2012): 3991-3998

11. McMurray Dunlop and Byrne. "The Photocatalytic Degradation of Atrazine on Nanoparticulate TiO2 Films," J. Photochem. Photobiol. A-Chem. (2006): 182 43-51.

12. Vamvakaki, Chaniotakis and Biosens. "Pesticide detection with a liposome-based nano-biosensor". Bioelectronics. 22. (2007): 2848-2853.
13. Torney, Trewyn, Lin and Wang. 'Mesoporous silica nanoparticles deliver DNA and chemicals into plants". Nat. Nanotechnol. 2. (2007): 295-300.

14. Nurkiewicz, Porter, Hubbs, Cumpston, Chen, Frazer and Castranova. "Nanoparticle inhalation augments particledependent systemic microvascular dysfunction". Part Fibre Toxicol. 5:1. (2008): doi:10.1186/1743-8977-5-1.

15. Monteiro-Riviere, Inman, Ryman-Rasmussen (2007). "Dermal effects of nanomaterials". In: Monteiro-Riviere, N.A., Tran, C.L. (Eds.), Nanotoxicology -Characterization, Dosing and Health Effects". Informa healthcare, New York, USA.

16. Tinkle, Antonini, Rich, Roberts, Salmen, DePree, and Adkins. "Skin as a route of exposure and sensitisation in chronic beryllium disease". Environ Health Perspect. 111(9): (2003): 1202-08.

17. Tsuji, Maynard and Howard. Research strategies for safety evaluation of nanomaterials, part IV: risk assessment of nanoparticles. Toxicol Sci 89(1). (2006): 42-50.

18. Radomski, Jurasz, Alonso-Escolano, Drews, Morandi, Malinski and Radomski. "Nanoparticleinduced platelet aggregation and vascular thrombosis". Brit J Pharmacol. 146(6). (2005): 882-93.

19. Elder, Lynch, Grieger, Chan-Remillard, Gatti, Gnewuch. "Human health risks of engineered nanomaterials: critical knowledge gaps in nanomaterials risk assessment”. In: Linkov, I., Steevens, J. (Eds.), Nanomaterials: Risks and Benefits. Springer, Dordrecht; (2009): p. 3- 29.

\section{Cite this article as:}

Khursheed Ahmad Wani and Richa Kothari.

Agricultural Nanotechnology: Applications and Challenges. Annals of Plant Sciences 7.3 (2018) pp. 2146 2148.

do http://dx.doi.org/10.21746/aps.2018.7.3.9 